\title{
Reconstitution and biochemical characterization of a new Pyridoxal-5'- phosphate biosynthetic pathway
}

\author{
Kristin E. Burns, Yun Xiang, Cynthia L. Kinsland, Fred W. McLafferty and Tadhg P. \\ Begley* \\ Department of Chemistry and Chemical Biology, Cornell University, Ithaca, NY 14853
}

\section{Supporting Information}

\section{Experimental}

General Information: All reagents were purchased from Sigma-Aldrich. UV-Visible spectra were obtained using a Hitachi-2010 spectrophotometer. NMR spectra were obtained with a Varian $600 \mathrm{MHz}$ spectrometer.

Cloning, Expression and Purification of YaaD and YaaE: B. subtilis yaaD and yaaE were cloned into pET-28a vectors, overexpressed in minimal media supplemented with glucose, and purified by Ni-Nta chromatography.

YaaD catalyzed isomerization of ribose-5-phosphate to ribulose-5-phosphate: YaaD $(1 \mathrm{mg})$ and ribose-5-phosphate $(3 \mathrm{mM})$ were combined in $515 \mu \mathrm{L}$ phosphate buffer $(50 \mathrm{mM}$, pD 7.8) in $\mathrm{D}_{2} \mathrm{O}$.

NMR of the product of PLP synthase: YaaD (3.8mg), YaaE (1.6mg), ribulose 5phosphate $(1 \mathrm{mM})$, dihydroxyacetone phosphate $(5 \mathrm{mM})$ and glutamine $(5 \mathrm{mM})$ were combined in $1.5 \mathrm{~mL}$ phosphate buffer $(50 \mathrm{mM}, \mathrm{pH} 8)$ and kept in the dark at $37^{\circ} \mathrm{C}$ overnight. Following alkaline phosphatase treatment $(0.5 \mathrm{mg})$ for 1.5 hours, the protein was removed and the sample was purified by HPLC and analyzed by NMR.

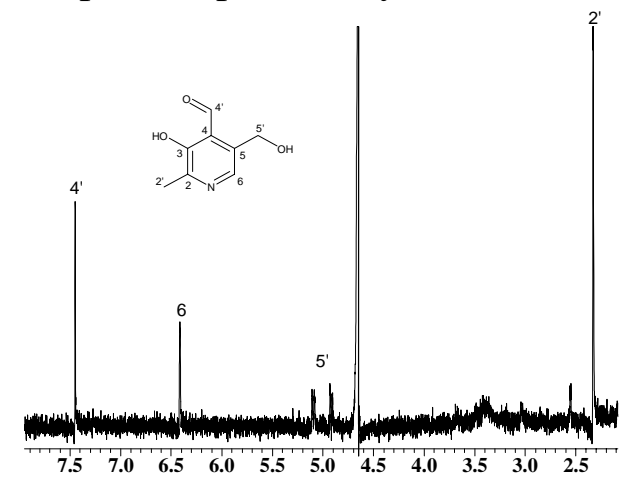

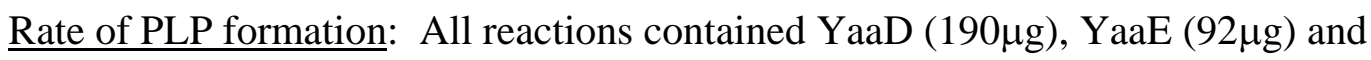
glutamine (6mM), with either ribose-5-phosphate or ribulose-5-phosphate (3mM), and dihydroxyacetone phosphate or glyceraldehyde-3-phosphate $(6 \mathrm{mM})$ in $350 \mu \mathrm{L}$ Tris $(50 \mathrm{mM}, \mathrm{pH} 8)$. The reactions were blanked against a sample without YaaD. 
Isomerization of dihyroxyacetone phosphate: $3 \mathrm{mM}$ dihydroxyacetone phosphate was added to $80 \mu \mathrm{g} \mathrm{YaaD}$ in $550 \mu \mathrm{L}$ phosphate buffer (pD 7.0) in $\mathrm{D}_{2} \mathrm{O}$ and NMR spectra were taken at various times. 